Z. Epileptol. 2021 · 34:249-250

https://doi.org/10.1007/s10309-021-00435-y

Angenommen: 30. Juni 2021

(c) Springer Medizin Verlag $\mathrm{GmbH}$, ein Teil von Springer Nature 2021

\section{Autoren}
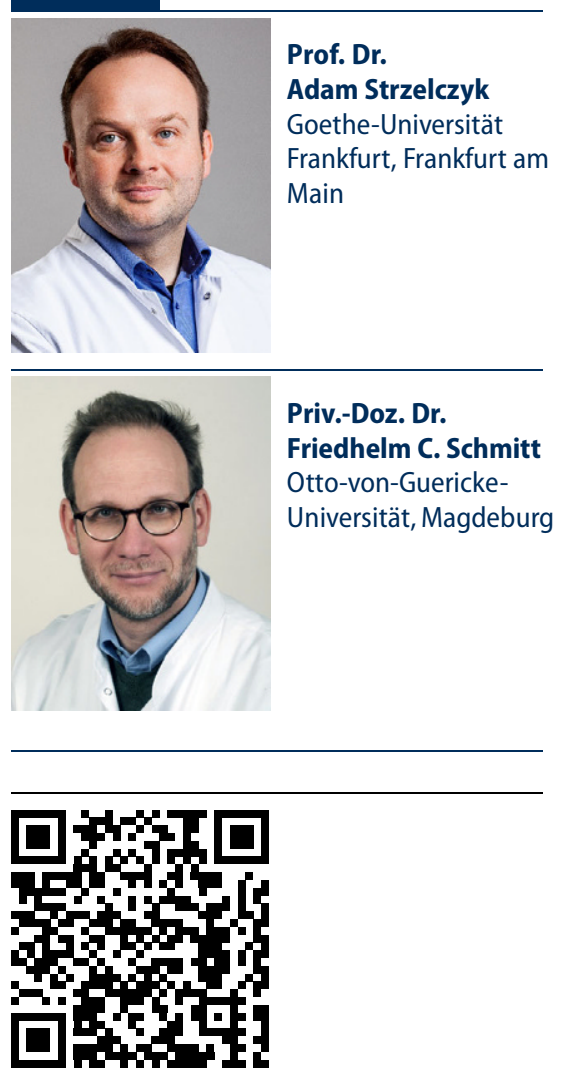

QR-Code scannen \& Beitrag online lesen

\title{
Langfristige Themenplanung und Aufruf zu Artikeln der Zeitschrift für Epileptologie
}

\author{
Adam Strzelczyk ${ }^{1}$ Friedhelm C. Schmitt ${ }^{2}$ \\ 'Epilepsiezentrum Frankfurt Rhein-Main, Zentrum der Neurologie und Neurochirurgie, Goethe- \\ Universität Frankfurt, Frankfurt am Main, Deutschland \\ ${ }^{2}$ Universitätsklinik für Neurologie, Otto-von-Guericke-Universität, Magdeburg, Deutschland
}

Gerade halten Sie das dritte Heft der Zeitschrift für Epileptologie des Jahres 2021 in Ihren Händen. Diesmal sind das Leitthema die „Telemedizin und mobile Gesundheitstechnologien"; die Gastherausgeber Frau Dr. Mues aus Bochum und Herr Prof. Surges aus Bonn haben mit sehr viel Engagement dieses Heft zu dem aktuell so wichtigen und dynamischen Thema für Sie zusammengestellt.

Mit diesem Herausgebereditorial möchten wir Sie auf zwei Änderungen hinweisen:

1. Wir möchten Sie nun vorab regelmäßig über unsere langfristige Themenhaftplanung bei der Zeitschrift für Epileptologie informieren: Denn so haben Sie als Leser und (hoffentlich) Manuskriptersteller die Möglichkeit, aktiv an der Themenwahl zu partizipieren. In • Tab. 1 finden Sie die aktuell vorgesehenen und in der Planung befindlichen Heftthemen für die nächsten ein bis zwei Jahre, und wir ermuntern Sie ausdrücklich, zu diesen Themen, aber auch zu anderen Themen spontan Artikel bei uns einzureichen. Mit dem Heft 1/2020 zu Temporallappenepilepsien [1] wurde die Reihe der Themenhefte mit den lokalisationsbezogenen Epilepsiesyndromen begonnen. Unser Ziel war es, auch einen Augenmerk auf Themen zu setzen, in denen epilepsiechirurgische Verfahren eine Rolle spielen. Diese Reihe findet im Heft 1/2023 mit dem Thema „Nichtläsionelle fokale Epilepsien" ihren Abschluss.

Wir möchten Sie aber auch ermuntern, Originalartikel, Übersichtsarbeiten und auch Fallberichte und historische Beiträge einzureichen, die nicht unbedingt mit ei- nem Themenheft in Verbindung zu setzen sind.

In diesem Heft finden Sie z.B. einen Fallbericht zum Einsatz der MR-gestützten stereotaktischen Laser-Thermoablation (sL-TA) bei einem Mädchen mit hypothalamischem Hamartom [2]. Während die sLTA in den Vereinigten Staaten bereits im Jahr 2011 eingeführt wurde [3], erfolgte eine CE-Zertifizierung in Europa erst 2018. Der erste Patient in Deutschland wurde mit der sL-TA allerdings erst im März 2019 behandelt [1, 4], u.a. weil einer breiten Anwendung in Deutschland die fehlende kostendeckende Durchführung der Prozedur entgegensteht. Die sL-TA ist bislang im DRG-System nicht abgebildet. Der in diesem Heft vorliegende Fallbericht belegt nun die Kosteneffektivität der Behandlung in diesem Einzelfall und kann somit helfen, die Methode und ihre Kosten gegenüber den Kostenträgern zu begründen. Mittlerweile wird dieses Verfahren in mehreren Zentren in Deutschland, Österreich und in der Schweiz bei Kindern und Erwachsenen eingesetzt. Übersichtsarbeiten zeigen, dass diese neue Methode eine schonende Therapieoption bei zahlreichen fokalen Epilepsiesyndromen [5-8] einschließlich der mesialen Temporallappenepilepsien darstellt $[5,7,9]$. Wir würden uns also auch freuen, wenn weitere Artikel zu neuen epilepsiechirurgischen Therapieoptionen eingereicht werden würden.

Aus Erlangen und Zürich erreichte uns ein historischer Beitrag [10] zu Berthold Kihn und dem Kihnschen Pulver. Die Kollegen haben sich sehr viel Mühe gegeben, die historischen Hintergründe und Patientenverläufe bis in die Gegen- 
Tab. 1 Langfristige Heftthemenplanung der Zeitschrift für Epileptologie bis 2024

\begin{tabular}{|l|l|l|}
\hline Heft & Thema & $\begin{array}{l}\text { Gastherausgeberinnen und } \\
\text { Gastherausgeber (Anmer- } \\
\text { kungen) }\end{array}$ \\
\hline Heft 4/2021 & Akut-symptomatische Anfälle & $\begin{array}{l}\text { E. Haberlandt, E. Pataraia } \\
\text { (Heftplanung abgeschlossen) }\end{array}$ \\
\hline Heft 1/2022 & $\begin{array}{l}\text { Frontallappenepilepsien und insuläre Epilepsi- } \\
\text { en }\end{array}$ & $\begin{array}{l}\text { M. Holtkamp, M. von Lehe, } \\
\text { F.C. Schmitt }\end{array}$ \\
\hline Heft 2/2022 & Im Alter beginnende Epilepsien & G. Krämer, F. von Podewils \\
\hline Heft 3/2022 & Menschen mit Behinderung und Epilepsie & F. Bösebeck, T. Dorn, F. Kerling \\
\hline Heft 4/2022 & Anfälle und EEG auf der Intensivstation & N.N. \\
\hline $\begin{array}{l}\text { Heftthemen } \\
2023\end{array}$ & $\begin{array}{l}\text { Nicht-läsionelle fokale Epilepsien } \\
\text { Geschlechterspezifische Aspekte in der Epilep- } \\
\text { tologie } \\
\text { Idiopathische fokale Epilepsien } \\
\text { Therapien jenseits von Antikonvulsiva }\end{array}$ & N.N. \\
\hline $\begin{array}{l}\text { Heftthemen } \\
\text { 2024 }\end{array}$ & \begin{tabular}{l} 
100 Jahre EEG und Hans Berger \\
\hline
\end{tabular} & F.C. Schmitt, A. Strzelczyk \\
\hline
\end{tabular}

wart zu ermitteln. Ein schöner Zufall war auch die inhaltliche Überschneidung einer spontanen Einreichung von Herrn Dr. Krämer aus Zürich („George Duncan Dawson [1912-1983] und seine Entdeckung der somatosensibel evozierten Riesenpotenziale bei progressiver Myoklonusepilepsie" [11]) mit dem Themenheft 1/2021 „Parietal- und Okzipitallappenepilepsien“ [12]. Unsere Rubrikherausgeber für historische Beiträge (Prof. Kasper aus Erlangen und Dr. Krämer aus Zürich) freuen sich sicher auf weitere neue Einreichungen von Ihnen.

2. Noch dieses Jahr wird die Einreichung von Manuskripten bei der Zeitschrift für Epileptologie auf den Editorial Manager umgestellt, um so den Autorinnen und Autoren, den Reviewern und den Herausgeberinnen und Herausgebern eine einfache Handhabung der Manuskripte zu ermöglichen. Wir denken, dass damit eine noch schnellere und zuverlässigere editorische Bearbeitung der Beiträge und deren Reviewingprozess gewährleistet ist. Wir sehen die Einführung des Editorial Managers auch als einen weiteren Schritt, die Zeitschrift für Epileptologie einer breiteren Leser- und Autorenschaft zur Verfügung zu stellen, und hoffen auch, dass es dadurch niedrigschwelliger zu einer spontanen Einreichung von Beiträgen kommt. In Kürze wird der Editorial Manager auf der Website des Springer-Verlages implementiert sein. Bis dahin bitten wir Sie, Artikel direkt an Frau Dr. S. Rehse (saskia.rehse@springernature.com) vom Springer-Verlag zu senden.

Wir freuen uns auf inhaltliche Anregungen, Lob und Kritik sowie auf Ihre Einreichungen.

Ihre
A. Strzelczyk
und
F.C. Schmitt

\section{Korrespondenzadresse}

Prof. Dr. Adam Strzelczyk

Epilepsiezentrum Frankfurt Rhein-Main, Zentrum der Neurologie und Neurochirurgie, Goethe-Universität Frankfurt

Schleusenweg 2-16, Haus 95, 60528 Frankfurt am Main, Deutschland

strzelczyk@med.uni-frankfurt.de

\section{Priv.-Doz. Dr. Friedhelm C. Schmitt}

Universitätsklinik für Neurologie, Otto-vonGuericke-Universität

Leipziger Str. 44, 39120 Magdeburg,

Deutschland

fc.schmitt@t-online.de

Interessenkonflikt. A. Strzelczyk und F.C. Schmitt geben an, dass kein Interessenkonflikt besteht.

\section{Literatur}

1. Baumgartner C, Strzelczyk A (2020) Update zu Temporallappenepilepsien.Z Epileptol 33:4-5

2. Kohlhase K, Strzelczyk A, Porto L, Beißel A, Curry DJ, Rosenow F, Schubert-Bast S (2021) MRgestützte stereotaktische Laserthermoablation eines hypothalamischen Hamartoms bei einer jungen Epilepsiepatientin: klinischer Verlauf und Kostenanalyse. Z Epileptol. https://doi.org/10. 1007/s10309-021-00415-2

3. Büntjen L, VogesJ,HeinzeHJ,Hinrichs H, SchmittFC (2017) Stereotaktische Laserablation. Z Epileptol 30:138-145

4. Schmitt $F C$, Büntjen L, Schütze $H$, Kaufmann J, Heinze HJ, Hinrichs $H$, Tempelmann C, Düzel $E$, Voges J (2020) Stereotaktische Laserthermoablation bei mesialer Temporallappenepilepsie mit Hippocampussklerose rechts - Patientenentscheidung, Durchführung und Visualisierung von Gedächtnisfunktion. ZEpileptol 33:42-49

5. Kohlhase K, Zöllner JP, Tandon N, Strzelczyk A, Rosenow F (2021) Comparison of minimally invasive and traditional surgical approaches for refractory mesial temporal lobe epilepsy: a systematic review and meta-analysis of outcomes. Epilepsia 62:831-845

6. Hoppe C, Helmstaedter C (2020) Laser interstitial thermotherapy (LiTT) in pediatric epilepsy surgery. Seizure 77:69-75

7. Ilse A, Büntjen L, SchmittFC (2017) Stereotaktische Laserablation für fokale Epilepsien. Z Epileptol 30:152-161

8. HoppeC, WittJA, HelmstaedterC,GasserT, Vatter H, Elger CE (2017) Laser interstitial thermotherapy (LiTT) in epilepsy surgery. Seizure 48:45-52

9. Kang JY, Sperling MR (2017) Laser-Thermoablation zur Behandlung von pharmakoresistenten Epilepsien.ZEpileptol 30:146-151

10. Kasper BS, Graf W, Lang JD, Krämer G (2021) Berthold Kihn und das Kihnsche Pulver. Z Epileptol. https://doi.org/10.1007/s10309-021-00416-1

11. Krämer G (2021) George Duncan Dawson (1912-1983) und seine Entdeckung der somatosensibel evozierten Riesenpotenziale bei progressiver Myoklonusepilepsie. Z Epileptol 34:108-110

12. Ramantani G, Stefan H (2021) Parietal- und Okzipitallappenepilepsien.Z Epileptol 34:4 